\title{
Micromachined Silicon-Based Analytical Microinstruments for Space Science and Planetary Exploration
}

\author{
F. J. Grunthaner, R. E. Stalder, S. Boumsellek, T. R VanZandt, T. W. Kenny, M. H. Hecht, \\ A. Ksendzov, M. L. Homer, R. W. Terhune, A. L. Lane, M. A. Butler*, and A. J. Ricco* \\ Center for Space Microelectronics, \\ Jet Propulsion Laboratory, California Institute of Technology, Pasadena, California, USA, 91109, \\ and *Sandia National Laboratories, Albuquerque, NM, USA, 87185-5800
}

For future planetary science missions, we are developing a series of microinstruments using the techniques of silicon-based micromachining. Conventional instruments such as chemical sensors, charged particle analyzers and mass spectrometers are reduced in size and effective volume to the dimension of cubic centimeters, while maintaining or enhancing performance. Using wafer/wafer bonding techniques, selective chemical etching, thin film growth, and high resolution lithography, complex three dimensional structures can be assembled. This paper discusses the design, implementation and performance of two new instruments: The Micromachined Bessel Box Auger Electron Spectrometer, and the Mars Soil Chemistry Experiment (MOx).

This work was surnnited by the United
States Depariment of Energy under
Contrist DE-AC $4.94 A$ L950no.

\section{Introduction}

The new financial realities facing the international space exploration effort strongly suggest that future space and planetary science programs will use robotic and instrumented probes which are smaller, lighter and less expensive than anything attempted in the last decade. Because cost is so strongly driven by launch weight, new planetary probes, including landed instruments, must be reduced to a total mass of 10 to 50 kilograms. This must include the power, control and communication subsystems together with the instrumental package. To meet this challenge, the latest technologies in solar and radiothermal power generation, computing and signal processing, and low noise communications must be utilized. To justify their cost, the scientific return of such missions must be significantly greater than those that have gone before. To go beyond the remote sensing which has characterized much of the existing space program, it is necessary to develop new microinstruments, which can measure many of the critical chemical and physical properties which are currently only accessible using earth-based laboratory analytical instrumentation,

In a new thrust at JPL, we are developing a series of microinstruments using the techniques of silicon-based micromachining. This approach takes conventional instruments such as chemical sensors, charged particle analyzers and mass spectrometers and reduces the size and effective volume to the dimension of cubic centimeters. Using wafer/wafer bonding techniques, selective chemical etching, thin film growth, and high resolution lithography, complex three dimensional structures can be assembled. Although the size and effective sample area are greatly reduced leading to a signal reduction, these microinstruments are readily organized in arrays. The net signal strength and throughput of the microinstrument array is quite competitive with a standard full size laboratory instrument.

In this paper, we will illustrate our approach by discussing the design, implementation and performance of two new instruments: The Micromachined Bessel Box Auger Electron Spectrometer, and the Mars '94 Soil Chemistry Experiment (MOx '94). In case of the Bessel Box spectrometer, we will discuss the electron optics of a one dimensional focusing implementation, compare calculated performance with measurement, and review the details of the multilayer $\mathrm{Si}$ wafer processing sequence. The MOx instrument is designed to measure Martian soil reactivity, addressing the existing models which suggest the existence of a strong oxidizing phase. This experiment places more than 65 different metal, semiconductor and organometallic thin films used as chemical sensors in contact with the Martian surface, and follows the solid state corrosion of these films over a period of more than 120 days, to establish the differential reactivity of the soil.

\section{MicroMachined Bessel Box}

Charged particle detectors are critical instruments for detection and energy analysis of ion and electron fluxes. These fluxes can be generated by a variety of processes and found in the immediate vicinity of planets, as well as in deep space. These instruments are also the key components of surface anzlytical measurements, such as Auger Electron Spectroscopy, and X-ray or UV Photoelectron Spectroscopy which could have a 


\section{DISCLAIMER}

This report was prepared as an account of work sponsored by an agency of the United States Government. Neither the United States Government nor any agency thereof, nor any of their employees, makes any warranty, express or implied, or assumes any legal liability or responsibility for the accuracy, completeness, or usefulness of any information, apparatus, product, or process disclosed, or represents that its use would not infringe privately owned rights. Reference herein to any specific commercial product, process, or service by trade name, trademark, manufacturer, or otherwise does not necessarily constitute or imply its endorsement, recommendation, or favoring by the United States Government or any agency thereof. The views and opinions of authors expressed herein do not necessarily state or reflect those of the United States Government or any agency thereof. 
strong impact on planetary chemical measurements. The electrostatic lenses which comprise charged particle optics are generally quite bulky, but are of simple shape and must be machined to stringent tolerances. In analyzing the performance of electrostatic optics, we have found that the dimensions of lens elements can be reduced to the order of 100's of micrometers, with corresponding reduction in path length. A serious reduction in the acceptance phase space results, but net throughput is actually enhanced by using arrays of such miniaturized elements. Our first prototype instrument to illustrate this concept is a simple bandpass energy filter based on a modified Bessel Box. The Bessel Box analyzer can be as simple as a can with two holes, one on the top and one on the bottom (representing the entrance and exit apertures) and a beam stop in the center of the device. 'In our implementation $^{2}$, the analyzer is assembled from a stack of silicon wafers. These wafers are machined with appropriate wet and dry chemical etchants to provide the necessary apertures, cavities and internal shapes required to form the required potential distribution. Thermally oxidized surfaces are used for insulators, and metallized wafers provide contacts and electrode surfaces. This is illustrated in Figure 1.

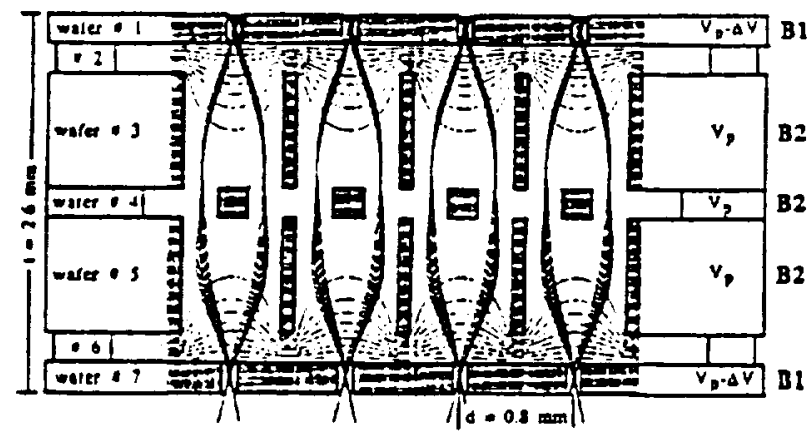

Fig.1 Cross section through the proturype array of four Bessel Boxes. The electrodes and spacers are made by a stack of seven anisotropically etched $\langle 110\rangle$ silicon wafers. Wafers 2 and 6 are insulating spacers, wafers 3,4 and 5 are electrically connected.

We have calculated the performance of the micro Bessel Box by ray-tracing using SIMION software ${ }^{3}$ for a specific set of voltages. These results for voltage and kinetic energy can be scaled linearly. In Figure 2(a) -2(c), cross sections of a single microlens are given together with ray-tracing results for electrons with different kinetic energies. Low-energy particles cannot pass the potential barrier and are reflected, whereas the deflection angle for high-energy particles is not sufficient to bend them around the axial beam stop into the exit pupil. The calculated performance of the prototype analyzer operating on input electrons of $101.3 \mathrm{eV}$, is estimated to give an energy bandwidth $(\Delta \mathrm{E})$ of $1.2 \mathrm{eV}$ full width at half maximum (FWHM). This is about $1 / 100$ of the barrier height with an applied voltage difference $\Delta \mathrm{V}$ of $\mathrm{V} / 10$. The energy resolution scales linearly with the barrier height, $\mathrm{V}$, and the voltage difference, $\Delta \mathrm{V}$.
The breakdown voltage of the thermal $\mathrm{SiO}_{2}$ is in the $\mathrm{keV}$ range, so high energy resolution can be achieved.
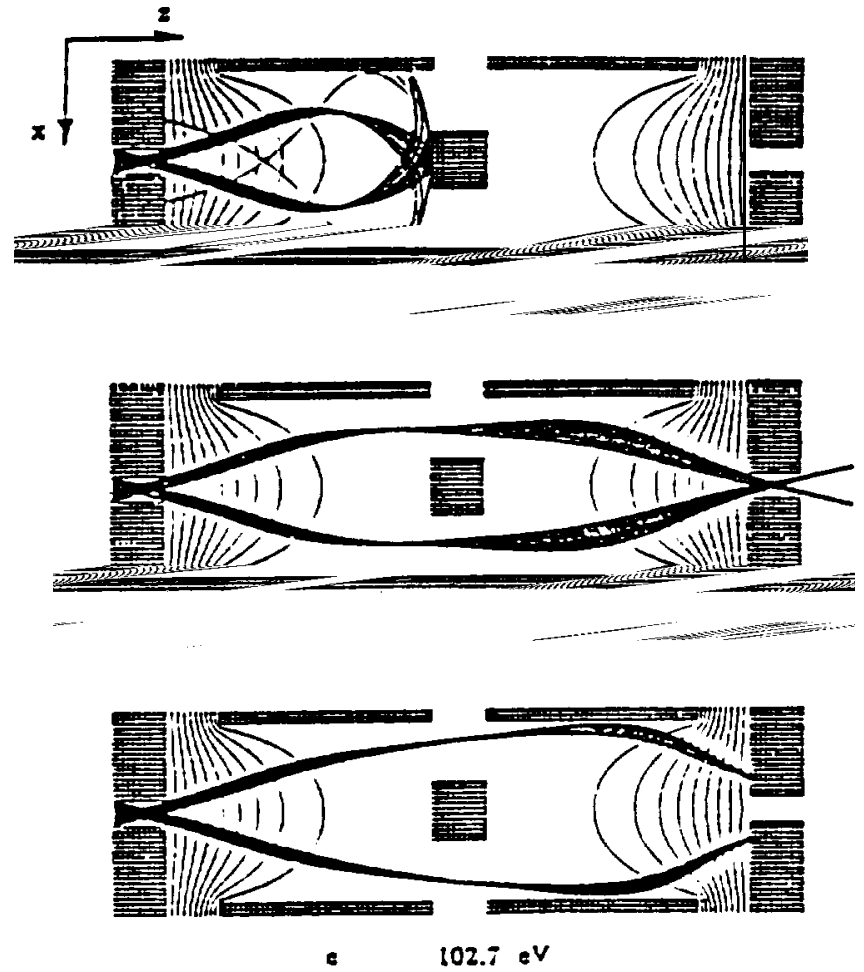

Figure 2(a) - (c). Ray-tracing results calculated with the SIMION program for an acceptance angle of $\pm 14^{\circ}$ and kinetic energies of $100.1,101.4$ and $102.7 \mathrm{eV}$, respectively.

We have also performed experimental tests with a shielded version of the micro Bessel Box. Slit widths of $100 \mu \mathrm{m}$. were used, and the analyzer was mounted between the sample and a secondary electron detector. An electron gun was used to excite samples of graphite and copper. The data for $1450 \mathrm{eV}$ primary electron energy, and a beam current of $100 \mathrm{nA}$, over an area of $1 \mathrm{~mm}$. diameter, is given in Figure 3.

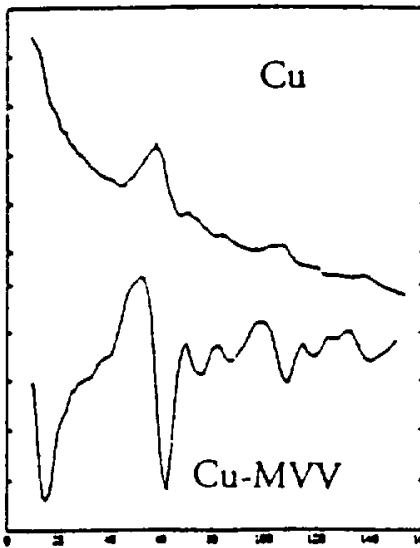

Kinetic Energy (eV)



Kinetic Energy (eV)
Figure 3. Auger Electron Spectra for copper (left panel) and graphite (right pancl). The integral spect. a are the upper curves, and derivative spectra are given in the lower curves. The carbon-KLL peak is found at $273.9 \mathrm{eV}$, and the copper-NIV peak at $59.9 \mathrm{eV}$. 
These results show that the current prototype gives an energy resolution of 1 to $3 \%$ and a transmission competitive with a $100 \mathrm{~mm}$. O.D. CMA. This simple implementation uses focusing in one dimension. Our ongoing effort is extending this concept to two dimensions, and initiating the application of these ideas to mats spectrometers, micro-optical systems and other physical instruments.

\section{Mars Soil Chemistry Experiment (MOx).}

The MOx experiment is designed to examine the oxidizing character of the Martian soil, by monitoring the oxidation or corrosion of a set of thin films to be placed in directly contact with the Martian surface. The chemical sensor head contains the complete experimental measurement system and consists of seven reaction heads, each of which is organized into twelve separate chemical cells. Of these seven heads, five are to be placed in contact with the Martian soil, and two are exposed to the atmosphere to measure gaseous reactions, and serve as controls for the soil contact results.

This instrument uses a precision optical reflectometer to measure changes in the reflectivity of thin film mirrors, whose thickness is in the range of $-100 \AA$. Oxidation or corrosion of these thin films changes their effective thickness, and the minimum detectable reflectivity change corresponds to oxidation of less than one monolayer, over a period of up to two years. To characterize soil reactivity, more than 18 different thin films, including metals, semiconductors, organometallic complexes, and simple carbon compounds, are simultaneously exposed and monitored. The inside of the sensor head is shown in Figure 4a, where the optical source is on the left, the line array detector assembly on the right, and the chemical sensor heads in the center. The optical source consists of two different wavelength LEDs, integrated into lightguide based optical bench. Fiber optic

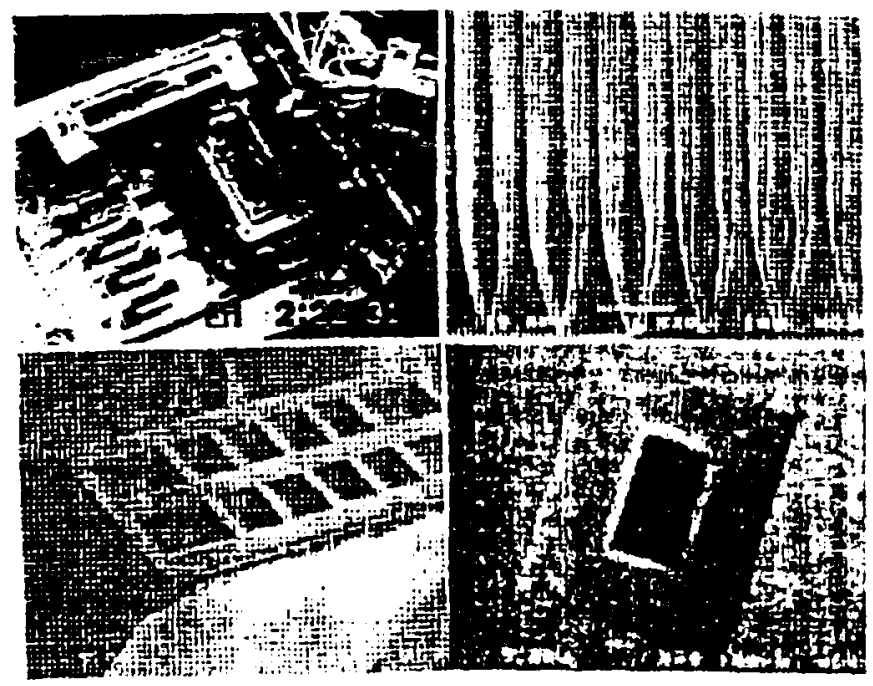

Figure 4. Upper left (a) shows vicw inco compleced scinsor head from top; upper right (b) shows micromachined fiber guides; lower lefe (c) shows fuson bonded chemical head and lower right (d) gives end on view of individual pixel of chemical cell, bundles bring the light from the light table to the sensor head and direct the reflected light from the thin film back to specific pixels on the detector array. This system is illustrated in Figure 5:

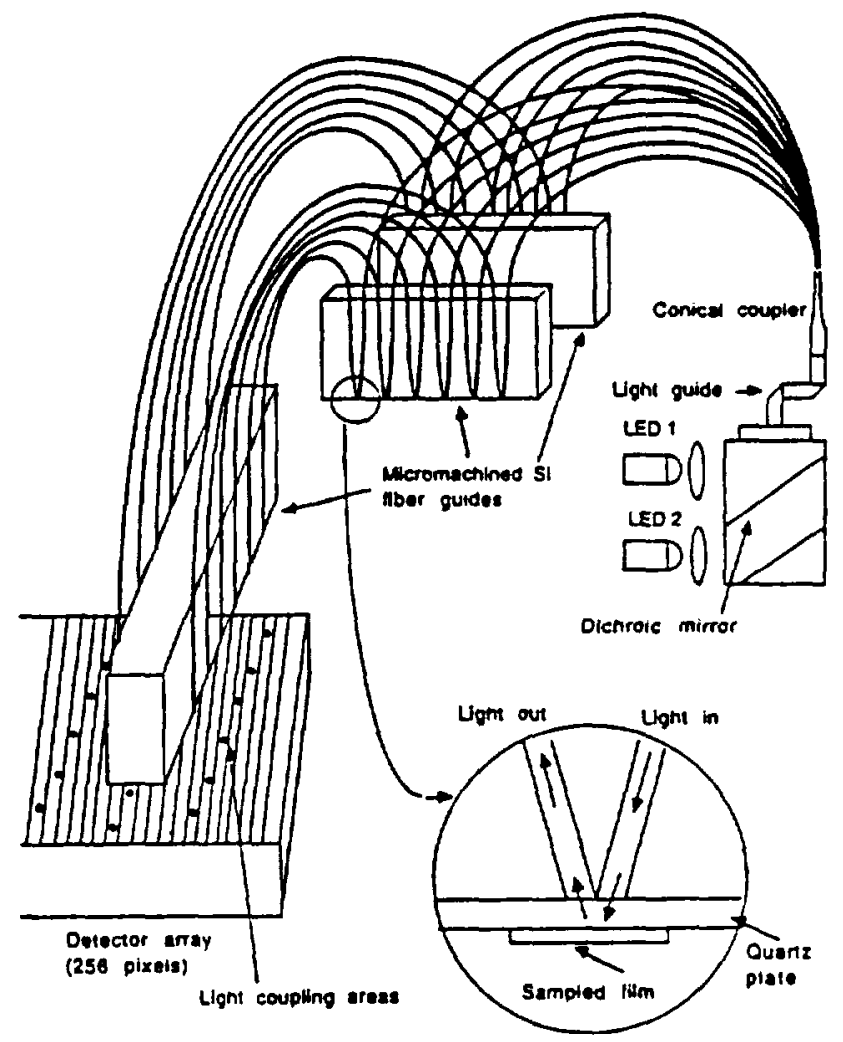

Figure 5. Overall system drawing of MOx sensor head. The eircled area shows detail of the reaction cell described in the text.

The reaction cell heads were fabricated by fusion bonding silicon and quartz wafers into a complex stack, which was then micromachined to form all of the chemical cells, Fig. 4c. The direct wafer bond approach eliminated chemical adhesives, from the reaction area. The guides for precise alignment of fibers to the reflector cell and the detector, Fig. 4b, were micromachined. This technique was also used to develop thin hermetic seals using $6000 \AA$ thick $a-S_{i} N_{4}$ films on a Si frame.

\section{Conclusions}

These microinstruments are based on the experimental understanding and chemical processing techniques entailed in silicon MOS processing, including interracial chemical reactions, ultraclean silicon surface preparation, hydrogen terminated silicon surface reactions, $\mathrm{SiO}_{2}$ defect reactions, silicide interface reactions and epitaxial film growth. Performance and future directions will be addressed in our presentation.

\section{References}

1) J. D. Allen et a1., Int. J. Mass Spec. Ion Phys. 8, 81 (1972). 2) R. E. Stalder et al., J. Vac. Sci. Tech. A 12(4), xxx (1994). 3) D. A. Dahl, J. E. Delmore, Idaho Natl. Eng. Lab., Idaho Falls, ID The research described in this paper was performed by the Center for Space Microelectronics Technology, Jet Propulsion Lab-oratory, California Institute of Technology and was sponsored by the National Aeronautics and Space Administration. 